\title{
Transcarotid approach for TAVI: an optimal alternative to the transfemoral gold standard
}

\author{
Pavel Overtchouk ${ }^{1}$, Ibrahim Alqdeimat ${ }^{1}$, Augustin Coisne ${ }^{1}$, Khalil Fattouch $^{2,3}$, Thomas Modine $^{1}$ \\ ${ }^{1}$ Interventional Cardiology and Cardiovascular Surgery Centre Hospitalier Regional, Universitaire de Lille (CHRU de Lille), Lille, France; \\ ${ }^{2}$ Department of Cardiovascular Surgery, GVM Care and Research, Maria Eleonora Hospital, Palermo, Italy; ${ }^{3}$ Department of Surgery and Cancer, \\ University of Palermo, Palermo, Italy \\ Correspondence to: Dr. Thomas Modine. Interventional Cardiology and Cardiovascular Surgery Centre Hospitalier Regional, Universitaire de Lille \\ (CHRU de Lille), 2 Avenue Oscar Lambret, Lille 59000, France. Email: thomas.modine@chru-lille.fr.
}

Submitted Jul 20, 2017. Accepted for publication Aug 14, 2017.

doi: $10.21037 /$ acs.2017.08.04

View this article at: http://dx.doi.org/10.21037/acs.2017.08.04

\section{Clinical vignette}

An 84-year-old male was addressed to our institution for degenerative stenosis of the aortic Carpentier-Edwards PERIMOUNT bioprosthesis (Edwards Lifesciences, Irvine, CA, USA). Transcatheter aortic valve implantation (TAVI) was favored to open surgery by the Heart Team because of patient's age, overweight (body mass index $31 \mathrm{~kg} / \mathrm{m}^{2}$ ), smoking, a history of moderate chronic renal failure [creatinine clearance with Modification of Diet in Renal Disease (MDRD) formula: $42 \mathrm{~mL} / \mathrm{min} / 1.73 \mathrm{~m}^{2}$ ], coronary artery disease, and a high STS score of $12.2 \%$. The preoperative coronary angiography showed no lesion; however aortic multidetector computed-tomography identified obstructive bilateral ilio-femoral arteriopathy that precluded trans-femoral pathway. Thus, the minimally invasive left trans-carotid access was deemed the most appropriate access for TAVI.

\section{Surgical techniques}

TAVI is usually performed in a hybrid operating room designed and equipped to perform both

open surgical and interventional procedures. The operating team usually includes an interventional cardiologist, a cardiac surgeon, a cardiac anesthesiologist, a radiology technician and two interventional assistants. At least one member of the team must be trained in preparation and loading of the transcatheter heart valve (THV) device, which is performed on the ancillary table while the surgeon and cardiologist access the aortic valve.
We chose a $23 \mathrm{~mm}$ Medtronic EVOLUT R (Medtronic, Minneapolis, MN, USA) THV to treat this stenosis of the $21 \mathrm{~mm}$ PERIMOUNT bioprosthesis, while the inner diameter is below $20 \mathrm{~mm}$.

The procedure was conducted under general anesthesia. Secondary percutaneous arterial right femoral access was achieved by insertion of a 5 -Fr vascular access sheath, whereby the pigtail catheter was inserted guided by a 0.035 " J-tipped soft guidewire (GuideRight ${ }^{\mathrm{TM}} 0.035^{\prime \prime} \times 260 \mathrm{~cm}$ length, tip $3 \mathrm{~mm}$ J-FS) and placed in the non-coronary cusp as a landmark and for aortography. A temporary pacemaker probe was inserted through the right femoral vein into the right ventricle.

After vertical $2-3 \mathrm{~cm}$ incision one or two fingers above the left clavicle, the common carotid artery was carefully dissected to avoid lesion of the vagus nerve (see Video 1: $2 \mathrm{~min} 20 \mathrm{~s}$ ). A complementary small incision $1 \mathrm{~cm}$ above the previous one grants stability to the sheath and the catheter during intravascular navigation (see Video 1: $3 \mathrm{~min} 30 \mathrm{~s}$ ). Intravenous heparin was administered to maintain an activated clotting time $\geq 250$ s. A 0.035 " J-tipped soft guidewire was used to guide the JR4 catheter (pigtail or AL1 catheters can be switched to, according to crossing difficulties) and then switched to a straight-tip guidewire to cross the aortic valve. The crossing step can be difficult and demands persistence, especially in case of degenerated bioprostheses (see Video 1: 5 min $26 \mathrm{~s}$ ). When the crossing was achieved, the catheter was pushed into the left ventricle, before switching the straight guidewire for a stiff guidewire (SAFARI pre-shaped TAVI guidewire 0.035 -inch $\times 300 \mathrm{~cm}$, Boston Scientific 
Marlborough, MA, USA or Amplatz extra stiff 0.035-inch guidewire, Cook, Inc., Bloomington, Indiana).

The EVOLUT R THV was loaded in the EnVeo R delivery system (Medtronic) in a sterile fashion. It was checked for proper orientation and seating. We made sure that contact was kept between the EVOLUT R stent in the sheath and the delivery catheter. The progression of the catheter through the carotid and the aorta was very smooth (see Video 1: $8 \mathrm{~min} 30$ s). Once properly positioned, the THV was deployed without the need for rapid pacing. Whenever the THV's position is deemed too low, the EVOLUT R can be recaptured and repositioned (see Video 1: $10 \mathrm{~min} 40 \mathrm{~s}$ ).

Absence of significant peri-prosthetic regurgitation was checked though aortography before carotid sheath removal (see Video 1: $11 \mathrm{~min} 40 \mathrm{~s}$ ). The carotid arterial access was then surgically repaired in a transversal fashion with PROLENE® Sutures 6-0, while short clamping proximally and distally to the vascular access (see Video 1: $13 \mathrm{~min} 30 \mathrm{~s}$ ). Post-operative trans-thoracic echocardiography confirmed the absence of periprosthetic leak, and carotid Dopplerechography showed no hematoma or fistula. Functional improvement was reported during follow-up.

\section{Comments}

TAVI has been developed to address the high morbimortality risk in the elderly patients presenting with degenerative aortic stenosis. These patients often have multiple comorbidities that preclude the optimal transfemoral approach; these include lower limb obliterative arterial disease with severe stenosis, vessel diameter $<6 \mathrm{~mm}$, heavily calcified vessels, tortuosity and significant descending aortic disease. The transcarotid vascular approach has been developed as an alternative. Preoperative multislice computed tomography shall confirm suitable supra-aortic anatomy through careful assessment of the ipsilateral common carotid (minimal luminal diameter $\geq 6 \mathrm{~mm}$ without significant, i.e., $\geq 50 \%$ stenosis or plaque at high risk of embolization), and the absence of subclavian, vertebral and contralateral carotid stenosis or occlusion, or congenital variants of the aortic arch (e.g., Bovine arch). Cerebral magnetic resonance angiography screening, if necessary, completed with a transcranial Doppler echography, shall evaluate the circle of Willis and collateral cerebral blood flow to identify patients with the potential for cerebral hypoperfusion. Intraoperatively, cerebral perfusion can be continually monitored using cerebral oximetry with near infrared spectrometry (Equanox 7600, Nonin Medical Inc., North Plymouth, Minnesota, USA).

The transcarotid approach should be performed by an experienced vascular surgeon due to the presence of important local structures, such as the vagus nerve and the respiratory tract: a lesion on the recurrent laryngeal nerve could cause dysphonia, and insufficient hemoptysis could lead to compressive hemoptysis and subsequent asphyxia. Post-operative care should include monitoring of signs of compressive hemoptysis (dysphonia, dysphagia, dyspnoea) and, whenever required an emergency orotracheal intubation is warranted, to provide time for a surgical revision.

Since its first description in 2010 (1), minimally-invasive surgery of the transcarotid pathway has been mastered by numerous Heart Teams, and recently it has been reported to have similar outcomes to transfemoral access in terms of mortality and morbidity (2). Our team reported a mortality of $6.3 \%$ at 30 days and $16.7 \%$ at 1 year. Furthermore, rates of 30-day cerebrovascular events were similar in our registry to existing data with transfemoral TAVI (3), in particular the rate of 30 -day stroke was under $2.5 \%$ with transcarotid TAVI $(2,4,5)$. Preference for local anesthesia with conscious sedation to general anesthesia is team-dependent; although our team reported possible improvement in neurological outcomes with local anesthesia and conscious sedation, most importantly regarding 30 -day strokes with a rate as low as $0 \%$ (versus $2.2 \%$ in patients treated under general anesthesia) (4). Transcarotid approach also allows for immediate ambulation and hospital stay as short as 4 days (5). Arguably, left carotid access for TAVI should be favored to the right because it provides superior coaxial alignment between the ascending aorta, optimal positioning for the trans-catheter valve during the device deployment and simple operating room configuration. However, right carotid access has also been used, with success (4). Furthermore, some teams use a femoral-carotid shunt to optimize neurological outcomes. To date there have been no randomized comparative studies that have compared the different approaches for TAVI, and CoreValve ${ }^{\circledR}$ (Medtronic) has been the most frequently used TAVI system for trans-carotid approach in published series $(2,4)$, although our practice has provided reassuring data using the Edwards-Sapien system (Edwards Lifesciences) as well.

\section{Acknowledgements}

None. 


\section{Footnote}

Conflict of Interest: Dr. Modine is a proctor and consultant for Medtronic and Microport. The other authors have no conflicts of interest to declare.

\section{References}

1. Modine T, Lemesle G, Azzaoui R, et al. Aortic valve implantation with the CoreValve ReValving System via left carotid artery access: first case report. J Thorac Cardiovasc Surg 2010;140:928-9.

2. Mylotte D, Sudre A, Teiger E, et al. Transcarotid Transcatheter Aortic Valve Replacement: Feasibility and

Cite this article as: Overtchouk $\mathrm{P}$, Alqdeimat I, Coisne A, Fattouch K, Modine T. Transcarotid approach for TAVI: an optimal alternative to the transfemoral gold standard. Ann Cardiothorac Surg 2017;6(5):555-557. doi: 10.21037/ acs.2017.08.04
Safety. JACC Cardiovasc Interv 2016;9:472-80.

3. Nombela-Franco L, Webb JG, de Jaegere PP, et al. Timing, predictive factors, and prognostic value of cerebrovascular events in a large cohort of patients undergoing transcatheter aortic valve implantation. Circulation 2012;126:3041-53.

4. Debry N, Delhaye C, Azmoun A, et al. Transcarotid Transcatheter Aortic Valve Replacement: General or Local Anesthesia. JACC Cardiovasc Interv 2016;9:2113-20.

5. Azmoun A, Amabile N, Ramadan R, et al. Transcatheter aortic valve implantation through carotid artery access under local anaesthesia. Eur J Cardiothorac Surg 2014;46:693-8; discussion 698 